\title{
Essential Role of the Metal Ion in the IPM-Assisted Domain Closure of 3-Isopropylmalate Dehydrogenase
}

Éva Gráczer ${ }^{\S}$, Peter V. Konarev", Tamás Szimler ${ }^{\S}$, András Bacsó $^{\S}$, Adrienn Bodonyi ${ }^{\S}$, Dmitri I. Svergun ${ }^{\# *}$, Péter Závodszky ${ }^{\S}$ and Mária Vas ${ }^{\S *}$

${ }^{\S}$ Institute of Enzymology, Biological Research Center, Hungarian Academy of Sciences, H-1518 Budapest, P. O. Box 7., Hungary

\#EMBL Outstation, c/o DESY, Notkestrasse 85, 22603 Hamburg, Germany

*Corresponding authors:

Mária Vas

Institute of Enzymology, BRC, Hungarian Academy of Sciences

H-1518 Budapest, P. O. Box 7., Hungary

Tel.: 361279 3152, Fax: 3614665465

E-mail: vas@enzim.hu

Dmitri Svergun

EMBL Outstation, c/o DESY

Notkestrasse 85, 22603 Hamburg, Germany

Tel.: 4940 89902/125

E-mail: svergun@embl-hamburg.de 


\begin{abstract}
X-ray structures of 3-isopropylmalate dehydrogenase (IPMDH) do not provide sufficient information about the role of metal-ion in the metal-IPM assisted domain closure. Here solution studies were carried out to test its importance. Small-angle X-ray scattering (SAXS) experiments with the Thermus thermophilus enzyme (complexes with single substrates) have revealed only a very marginal (0-5\%) extent of domain closure in the absence of the metalion. Only the metal-IPM complex, but neither the metal-ion nor the free IPM itself, is efficient in stabilizing the native protein conformation as confirmed by denaturation experiments with Escherichia coli IPMDH and by studies of the characteristic fluorescence resonance energy transfer (FRET) signal (Trp to bound NADH) with both IPMDHs. A possible atomic level explanation of the metal-effect is given.
\end{abstract}

\title{
ABBREVIATIONS
}

IPMDH: 3-isopropylmalate dehydrogenase (EC 1.1.1.85)

IPM: threo-D -3-isopropylmalate

MnIPM: complex of IPM with $\mathrm{Mn}^{2+}$

MgIPM: complex of IPM with $\mathrm{Mg}^{2+}$

EDTA: Ethylene Diamine Tetraacetic Acid

SAXS: small angle X-ray scattering

Tt: Thermus thermophilus

Ec: Escherichia coli

$\mathrm{Nbs}_{2}$ : Ellman's reagent [5,5'-dithiobis(2-nitrobenzoic acid)]

FRET: fluorescence resonance energy transfer 
*Highlights

Keywords:

Domain movement, Metal effects, 3-isopropylmalate dehydrogenase, small-angle X-ray scattering 


\section{INTRODUCTION}

Relative movement of protein domains, as a manifestation of the conformational flexibility (cf. reviews [1-3]) is frequently involved in enzyme mechanisms. There are examples of domain closure even among the thermostable enzymes that are known to possess a less flexible structure, such as 3-isopropylmalate dehydrogenase (IPMDH) from a thermostable bacterium, Thermus thermophilus $(T t)$. The recently published crystal structures of the various substrate complexes of the dimeric $T t$ IPMDH have revealed the relative movement of the two domains within each subunit and allowed identification of the two most important hinges [4]. It is highly probable that this domain closure assures the proximity and the optimal environment for the two reacting substrates and this property is characteristic for all IPMDHs, independent of their origin or of their thermostability. Accordingly, the crystal structure of Thiobacillus ferrooxidans IPMDH, a mesophilic form in its complex with MgIPM exhibits the closed conformation that is possibly the catalytically competent form.

However, the structural principles that govern protein domain motions in terms of side-chain and backbone interactions, as well as the role of substrates, in mediating such motions are generally poorly understood. In case of IPMDH, based on the crystal structures, hypotheses have been deduced about the role of the substrates in domain closure. Namely, the effects on both main hinges could be attributed to MnIPM and could be described in atomic details [4]. This hypothesis is in agreement with the protecting effect of MnIPM, but neither NAD nor NADH, against protein denaturation as observed in solution experiments with either $T t$ or Escherichia coli (Ec) IPMDHs [5]. On the other hand, enzyme kinetic experiments have shown complete loss of activity upon withdrawal of the metal-ion by addition of EDTA, even if IPM and NAD were present [6,7]. These works on one side may indicate an essential role of the metal-ion in the chemical catalysis and on the other side, raises the possibility of the 
importance of the metal in assisting formation of the active conformation. In order to answer the latter question structural information about IPMDH in the absence of the metal-ion would be required. However, at present only the apoenzyme X-ray structure is available for the metal-free IPMDH.

In the present work solution studies (denaturation-renaturation, FRET and SAXS; cf. abbeviations) experiments have been carried out in order to test an assumed effect of the metal-ion on the enzyme conformation. The importance of solution experiments are always underlined, even if crystal structures exist, since large scale domain movements may be hindered by lattice forces.

\section{MATERIALS AND METHODS}

\section{Enzyme and chemicals}

Wild type $T t$ and $E c$ IPMDHs were expressed in Ec BL21 DE3 pLysS strain, purified and stored as described previously [5,8]. Threo-DL-3-isopropylmalic acid (IPM) was

purchased from Wako Biochemicals (Japan), NAD and NADH were Sigma products. Ellman's reagent (Nbs2) was obtained from Serva. All other chemicals were commercially available high purity grade products.

\section{Enzyme activity measurements}

Activity of IPMDH (6-12 $\mu \mathrm{g} / \mathrm{mL}$, i.e., $0.16-0.32 \mu \mathrm{M}$ monomer) was assayed in the presence of $0.5 \mathrm{mM}$ IPM, $0.5 \mathrm{mM} \mathrm{MnCl}_{2}, 2 \mathrm{mM} \mathrm{NAD}$ and $10 \mathrm{mM}$ DTT in $25 \mathrm{mM}$ MOPS/KOH buffer (pH 7.6). Formation of NADH was recorded spectrophotometrically at 
$340 \mathrm{~nm}$ at $20{ }^{\circ} \mathrm{C}$ using a Jasco (Tokyo, Japan) V-550 spectrophotometer equipped with a Grant Y6 thermostat. The molar activities of $T t$ and $E c$ IPMDH subunits were $238 \pm 30$ and $700 \pm 80 \mathrm{~min}^{-1}$, respectively. When $\mathrm{MgCl}_{2}(2 \mathrm{mM})$ replaced $\mathrm{MnCl}_{2}$ the molar activities of $T t$ and $E c$ IPMDHs were $135 \pm 20$ and $330 \pm 40 \mathrm{~min}^{-1}$, respectively.

Control kinetic experiments with EDTA indicated that EDTA does not compete with binding of IPM, i.e. inhibition by EDTA is solely due to withdrawal of the metal-ion from the IPMDH active site.

A much weaker binding of metal-free IPM to IPMDH compared to MnIPM could be deduced from the slight inhibitory phenomenon observed when IPM was applied in molar excess over the metal-ion (not presented).

\section{FRET experiments}

The fluorescence resonance energy transfer (FRET) between Trp(s) of IPMDH and the bound NADH was recorded at $20{ }^{\circ} \mathrm{C}$ in the presence of MgIPM as reported by Dean and Dvorak [9] using a SPEX Fluoromax-3 spectrofluorimeter equipped with a Peltier thermostat (Edison, NJ). The usual mixture contained $12 \mu \mathrm{g} / \mathrm{mL}(0.32 \mu \mathrm{M}$ monomer) IPMDH, $12.5 \mu \mathrm{M}$ NADH, $3 \mathrm{mM} \mathrm{MgCl}$ and $0.5 \mathrm{mM}$ IPM. In the absence of $\mathrm{MgCl}_{2} 1 \mathrm{mM}$ EDTA was also present. The protein was excited at $295 \mathrm{~nm}$ and the emission by the bound NADH was recorded between 300 and $550 \mathrm{~nm}$ in a cuvette with $10 \mathrm{~mm}$ path length. The slits of 2 and 4 nm were applied for excitation and emission, respectively. 
Denaturation of Ec IPMDH was initiated by dilution into a solution containing $8.5 \mathrm{M}$ urea, $25 \mathrm{mM}$ MOPS/KOH buffer ( $\mathrm{pH}$ 7.6). Time courses of unfolding were followed at $20{ }^{\circ} \mathrm{C}$ by measuring either protein fluorescence or thiol-reactivity. The protein concentration in the denaturation experiments varied from 12 to $425 \mu \mathrm{g} / \mathrm{mL}$ (from 0.32 to $11.2 \mu \mathrm{M}$, monomer). All experiments were carried out both in the absence and in the presence of substrates.

When denaturation was followed by detecting protein fluorescence the effect of IPM both with and without the metal-ion (in the latter case EDTA was present) have been investigated. In the denaturation mixtures $1 \mathrm{mM}$ IPM and $1 \mathrm{mM} \mathrm{MnCl}_{2}$ or $10 \mathrm{mM}$ IPM and $10 \mathrm{mM}$ EDTA were applied. The mixtures also contained $2 \mathrm{mM}$ DTT in order to prevent oxidation of the thiols liberated during denaturation. The samples were excited at $275 \mathrm{~nm}$, and the emission was monitored at $335 \mathrm{~nm}$ using a cuvette with $10 \mathrm{~mm}$ path length. The slits of 2$4 \mathrm{~nm}$ were applied for excitation and emission.

Denaturation of Ec IPMDH could be also followed by measuring the protein thiolgroups liberated during unfolding. This enzyme contains 6 thiol groups per monomer and only one is reactive under native conditions. The remaining thiols are buried in the tertiary structure, but become reactive upon denaturation. In these experiments (that had to be carried out in the absence of DTT) only 4-5 mol thiols/mol enzyme monomer have been detected using a molar absorption coefficient $\left(\varepsilon_{412}\right)$ of $14150 \mathrm{M}^{-1} \mathrm{~cm}^{-1}$ [10], due to their partial oxidation. The time course of denaturation was recorded by detecting the thiol-modification at high concentration of $\mathrm{Nbs}_{2}(240 \mu \mathrm{M})$ that ensured a much faster blocking of the liberated thiols compared to the rate of denaturation. During denaturation, when applied, both IPM and $\mathrm{MnCl}_{2}$ were $1 \mathrm{mM}$, while $\mathrm{NAD}(\mathrm{H})$ and EDTA were 3 and $10 \mathrm{mM}$, respectively. 
Synchrotron radiation X-ray scattering data were collected on the X33 beam line [11] at the Hamburg EMBL Outstation (on the DORIS III storage ring, at DESY). Solutions of wild type $T t$ IPMDH, its complexes with MnIPM, MgIPM, Mn plus NAD, Mn plus NADH and with NAD plus MnIPM (functioning complex) and NADH plus MnIPM (non-functioning complex), both in the absence and presence of $10 \mathrm{mM}$ EDTA (in the latter case without addition of metal-ion) in $25 \mathrm{mM} \mathrm{MOPS/KOH}$ buffer, $\mathrm{pH} 7.6$, measured at protein concentrations in the range from 5.0 to $10 \mathrm{mg} / \mathrm{ml}$ using pixel $1 \mathrm{M}$ PILATUS detector (DECTRIS, Switzerland) at a sample-detector distance of $2.7 \mathrm{~m}$, and wavelength $\lambda=1.5 \AA$, covering the momentum transfer range $0.012<s<0.55 \AA^{-1}(\mathrm{~s}=4 \pi \sin (\theta) / \lambda$ where $2 \theta$ is the scattering angle). The concentrations of $\mathrm{Mn}^{2+}, \mathrm{Mg}^{2+}, \mathrm{IPM}, \mathrm{NAD}$ and NADH in the protein samples were $1 \mathrm{mM}, 10 \mathrm{mM}, 0.5 \mathrm{mM}, 2.5 \mathrm{mM}$ and $2.5 \mathrm{mM}$, respectively, except that much higher concentration $(10 \mathrm{mM})$ of IPM was applied in the presence of EDTA due to the possible weakening of IPM binding in the absence of the metal-ion (cf. Methods).

The effect of EDTA on the apoenzyme conformation was checked in a separate SAXS experiment. It was also tested by activity measurements that EDTA does not interfere with binding of IPM.

No measurable radiation damage was detected by comparison of eight successive time frames with 15 second exposures. The data were averaged after normalization to the intensity of the incident beam, corrected for the detector response, and the scattering of the buffer was subtracted. The difference data were extrapolated to zero solute concentration following standard procedures. All data manipulations were performed using the program package PRIMUS [12].

The forward scattering $\mathrm{I}(0)$ and the radius of gyration $R_{\mathrm{g}}$ were evaluated using the Guinier approximation [13] assuming that at very small angles $\left(s<1.3 / R_{\mathrm{g}}\right)$ the intensity is represented as $\mathrm{I}(s)=\mathrm{I}(0) \exp \left(-\left(s R_{\mathrm{g}}\right)^{2} / 3\right)$. These parameters were also computed from the entire 
scattering patterns using the indirect transform package GNOM [14]. The molecular masses of the solutes, evaluated by comparison of the forward scattering with that from reference solutions of bovine serum albumin, were compatible with the dimeric state of the enzyme for all samples.

The radii of gyration and the scattering patterns from the crystallographic models of wild type $T t$ IPMDH and its complexes were computed using the program CRYSOL [15]. Given the atomic coordinates, the program fits the experimental intensity by adjusting the excluded volume of the particle and the contrast of the hydration layer to minimize the discrepancy, defined by $\chi^{2}$ :

$$
\chi^{2}=\frac{1}{N-1} \sum_{j}\left[\frac{I_{\text {exp }}\left(s_{j}\right)-c I_{c a l c}\left(s_{j}\right)}{\sigma\left(s_{j}\right)}\right]^{2}
$$

where $N$ is the number of experimental points, $c$ is a scaling factor, $I_{\text {exp }}\left(s_{j}\right)$ and $I_{\text {calc }}\left(s_{j}\right)$ are the experimentally determined and calculated intensities, respectively; and $\sigma\left(s_{j}\right)$ the experimental error at the momentum transfer $s_{j}$.

The program OLIGOMER [12] was used to calculate the ratio of open-form and closed-form species present in $T t$ IPMDH solutions. The experimental intensity $I_{\text {exp }}(s)$ was represented by linear combinations of the curves computed from the open and closed crystallographic models of $T t$ IPMDH. Given the scattering curves of such components, the program OLIGOMER finds the volume fractions of each component by solving a system of linear equations to minimize the discrepancy between the experimental and calculated scattering curves.

\section{RESULTS and DISCUSSION}




\section{The Metal-ion Dependence of the FRET Phenomenon}

It has been noted that spectral changes, characteristic of FRET from the Trp side chain(s) of IPMDH to the bound NADH can occur but, interestingly only if the substrate IPM is also bound [9]. Here we show that only the metal-complex of IPM is effective in this respect. The spectra in Figs. 1A and 1B illustrate the FRET phenomenon in the cases of $T t$ and Ec IPMDHs, respectively: a new emission band of the NADH (acceptor) bound to IPMDH around $410 \mathrm{~nm}$ appeared with a concomitant decrease of the emitted protein (Trp) fluorescence (donor) around $340 \mathrm{~nm}$, upon addition of IPM to the binary complex of IPMDH and NADH in the presence of the metal-ion (here $\mathrm{Mg}^{2+}$ ). However, no emission band is appeared even if high excess $(10 \mathrm{mM})$ IPM was added to the mixture in the presence of EDTA, a known metal-complexing agent. Afterwards, addition of the metal in stochiometric excess compared to EDTA led to the reappearence of the emission characteristic of FRET. The experimental results were qualitatively the same if $\mathrm{Mn}^{2+}$ was added in place of $\mathrm{Mg}^{2+}$. The only notable difference is a somewhat smaller intensity of the FRET-band in the presence of $\mathrm{Mn}^{2+}$ compared to $\mathrm{Mg}^{2+}$. On the other hand, if only the metal-ion was present (without addition of IPM) the emission band characteristic of FRET did not appear at all. This experiment clearly indicates that only the metal-IPM complex and neither IPM nor the metalion alone is responsible for the FRET phenomenon.

It is well-known that Trp and NADH are a good donor-acceptor pair with characteristic distance $R_{0} \approx 25 \AA$ for $50 \%$ efficiency of energy transfer in case of favourable orientation of their electronic dipoles [16]. From the known crystal structure of $T t$ IPMDH in complex with MnIPM it could be deduced that the protein conformational changes accompanying MnIPM binding optimize both position and orientation of the Trp side chains relative to the bound coenzyme. From mutational studies with the Tt enzyme W77 and W195 
could be identified as the participants in the FRET process. A reasonable structural explanation has been also provided: it was concluded that while the FRET signal of W77 reports on formation of the domain-closed conformation, that of W195 reports on the structural changes occurring at both subunit-subunit and domain-domain interfaces upon binding of IPM [8]. It is possible that the same type of mechanisms operate also in case of $E c$ enzyme where the equivalent side chains are W81 and W205 [17]. Our present experiment indicates that these conformational events are solely due to binding of the metal-IPM complex, i.e. do not be take place upon binding of the metal-free IPM.

\section{Specific Protection against Denaturation by the Metal-IPM Complex}

Substrates binding, in addition to the effect of stabilising the catalytically competent protein conformation (e.g. the domain-closed form of IPMDH), in general, can also stabilize the protein structure against damage caused by denaturating agents. We have reported that binding of MnIPM markedly reduces the rates of unfolding of IPMDHs. This effect is more prominent for the less stable enzyme variants and it is only marginal in case of the thermostable $T t$ IPMDH, but much more pronounced in the cases of the mesophilic $E c$ and the psychrotrophic Vibrio sp. I5 IPMDHs [5]. Here we repeated the denaturation experiment with Ec IPMDH in the presence of either MnIPM or metal-free IPM. In the latter case EDTA was added to the denaturation mixture in order to remove the metal-ion contaminations of the buffer or urea solutions. It is clear from Fig. 2A that the rate of denaturation is significantly slowed down in the presence of MnIPM and that this protecting effect is completely abolished in the presence of EDTA (i.e. in the absence of the metal-ion). It is also notable that EDTA itself has no any effect on the rate of denaturation. Thus, protection of IPMDH structure 
against denaturation is solely due to binding of the metal-complex of IPM (MnIPM) and neither the metal-ion itself nor the metal-free IPM was effective at all.

Similar results have been obtained when denaturation of Ec IPMDH was followed by measuring the time course of liberation of the reactive thiol-groups upon incubation is $8 \mathrm{M}$ urea (Fig. 2B). It is seen that the native state $E c$ IPMDH already possesses 1 mole reactive thiols per subunit. Under our experimental conditions this reactive thiol reacts faster than the rate of denaturation, i.e. its reaction is practically finished before occurrence of appreciable extent of denaturation. The effects of various substrates on the rate of denaturation of $E c$ IPMDH obtained by the two different methods are summarised in Table 1 . The results clearly demonstrate the importance of the metal in respect of the conformational effect of MnIPM. On the other hand, the substrates NAD and NADH have no any meaningful protecting effects against denaturation, even in the presence of the metal-ion.

\section{Conformational Changes of $T t$ IPMDH upon Substrate Binding as Detected by SAXS}

SAXS is the most appropriate method to test the changes of protein conformation occurring in solution, especially if it is accompanied by changes in the shape of the molecule that can indeed be assumed when domain closure is taken place.

In case of IPMDH a domain-closed conformations have been determined from the crystal structures of both $T f$ [18] and $T t$ [4] IPMDHs in complexes with MgIPM and MnIPM, respectively. Furthermore, no appreciable domain closure have been detected in the crystal structures of apoenzyme as well as in complexes of $T t$ IPMDH with either NAD [19] or NADH [4]. Up to now, however, no crystal structures of the complexes with both MnIPM and $\mathrm{NADH}$ and any of the metal-free complexes have been yet published. 
In the present SAXS measurements we not only tested the appearance of the crystallographically detected conformations in solutions, but systematically investigated all the possible enzyme-ligand complexes of $T t$ IPMDH. The experimental scattering curves of the various complexes of $T t$ IPMDH in the presence and in the absence of the metal-ions $\mathrm{Mn}^{2+}$ or $\mathrm{Mg}^{2+}$ are shown in Figs. 3A and 3B, respectively. Table 2 summarizes the calculated $R \mathrm{~g}$ values as well as the extent of domain closure as expressed by percentage of the open and closed forms estimated by the way described in the Methods. These data are only qualitatively consistent with the results obtained in a previous SAXS study with $T t$ IPMDH, in which 1 $\mathrm{mM} \mathrm{Mg}^{2+}$ has been applied [20]. In that work partial domain closure has been concluded for the complexes with either NADH or IPM, while the conformation of the ternary complex with both IPM and NADH has been interpreted as a completely closed one.

Our SAXS data support at least partial occurrence of domain closure in the MnIPM and in the stable (non-functioning) MnIPM plus NADH containing complexes. However, surprisingly, the conformational equilibrium of the functioning MnIPM plus NAD containing complex is apparently shifted towards the open form. This phenomenon could be commented in light of the view that an efficient active enzyme might spend only a little time in the closed conformation. Furthermore, the data unequivocally demonstrate the essential role of the metal-ion in complexing with IPM: domain closure takes place exclusively upon binding of metal-IPM and the metal-free IPM is found not to be effective.

From the existing crystal structure of the MnIPM complex of $T t$ IPMDH one can make a proposal about the essential role of the metal-ion in assuring the stereospecific substrate-like binding of IPM. Fig. 4 indeed illustrates the proper orientation of the OH-group of IPM by the complexing $\mathrm{Mn}^{2+}$-ion. At the same time the two $\mathrm{COOH}$-groups of IPM are liganded by three arginines (R94, R104 and R132) that are positioned at about equal distances (3.5-4.0 $\AA$ ) from each other. In the absence of the metal-ion the positions of the two carboxyls might be 
exchanged without changing their interactions with the arginines. However, in this alternative binding mode the $\mathrm{OH}$-group would not be positioned appropriately for occurrence of the redox process. Furthermore, in the absence of the $\mathrm{Mn}^{2+}$ mediated connection of IPM with D241 and D245 (belonging to $\alpha$-helix h) occurrence of domain closure (promoted by the movement of $\alpha$-helix h) would also be prevented. In order to test this hypothesis further crystallographic work with $T t$ IPMDH complexed with the metal-free IPM are in progress in our laboratory.

\section{ACKNOWLEDGEMENTS}

The financial support by the grants OTKA (NK 77978) of the Hungarian National Research Fund is gratefully acknowledged. We would also like to thank the synchrotron facilities EMBL (Hamburg Outstation, Germany) at the beamline at the DORIS storage ring, DESY. The research leading to these results has received funding from the European Community's Seventh Framework Programme (FP7/2007-2013) under grant agreement No. 226716. 


\section{REFERENCES}

[1] Gerstein, M. and Echols, N. (2004) Exploring the range of protein flexibility, from a structural proteomics perspective. Curr Opin Chem Biol 8, 14-9.

[2] Henzler-Wildman, K.A., Lei, M., Thai, V., Kerns, S.J., Karplus, M. and Kern, D. (2007) A hierarchy of timescales in protein dynamics is linked to enzyme catalysis. Nature 450, 913-6.

[3] Vas, M., Varga, A. and Gráczer, E. (2010) Insight into the mechanism of domain movements and their role in enzyme function: example of 3-phosphoglycerate kinase. Curr Protein Pept Sci 11, 118-47.

[4] Gráczer, É., Merli, A., Singh, R.K., Karuppasamy, M., Závodszky, P., Weiss, M.S. and Vas, M. (2011) Atomic level description of the domain closure in a dimeric enzyme: Thermus thermophilus 3-isopropylmalate dehydrogenase. Mol Biosyst 7, 1646-59.

[5] Gráczer, É., Varga, A., Hajdú, I., Melnik, B., Szilágyi, A., Semisotnov, G., Závodszky, P. and Vas, M. (2007) Rates of unfolding, rather than refolding, determine thermal stabilities of thermophilic, mesophilic, and psychrotrophic 3-isopropylmalate dehydrogenases. Biochemistry 46, 11536-49.

[6] Yamada, T., Akutsu, N., Miyazaki, K., Kakinuma, K., Yoshida, M. and Oshima, T. (1990) Purification, catalytic properties, and thermal stability of threo-Ds-3isopropylmalate dehydrogenase coded by leuB gene from an extreme thermophile, Thermus thermophilus strain HB8. J Biochem (Tokyo) 108, 449-56.

[7] Wallon, G., Yamamoto, K., Kirino, H., Yamagishi, A., Lovett, S.T., Petsko, G.A. and Oshima, T. (1997) Purification, catalytic properties and thermostability of 3isopropylmalate dehydrogenase from Escherichia coli. Biochim Biophys Acta 1337, 105-12. 
[8] Gráczer, E., Varga, A., Melnik, B., Semisotnov, G., Závodszky, P. and Vas, M. (2009) Symmetrical refolding of protein domains and subunits: example of the dimeric twodomain 3-isopropylmalate dehydrogenases. Biochemistry 48, 1123-34.

[9] Dean, A.M. and Dvorak, L. (1995) The role of glutamate 87 in the kinetic mechanism of Thermus thermophilus isopropylmalate dehydrogenase. Protein Sci 4, 2156-67.

[10] Riddles, P.W., Blakeley, R.L. and Zerner, B. (1979) Ellman's reagent: 5,5'-dithiobis(2nitrobenzoic acid)--a reexamination. Anal Biochem 94, 75-81.

[11] Roessle, M.W. et al. (2007) Upgrade of the small-angle X-ray scattering beamline X33 at the European Molecular Biology Laboratory, Hamburg. J. Appl. Cryst. 40 (Supplement), s190-s194.

[12] Konarev, P.V., Volkov, V.V., Sokolova, A.V., Koch, M.H.J. and Svergun, D.I. (2003) PRIMUS: a Windows PC-based system for small-angle scattering data analysis. J. Appl. Crystallogr. 36, 1277-1282.

[13] Guinier, A. (1939) La diffraction des rayons X aux trés petits angles; application á l'étude de phénoménes ultramicroscopiques. Ann. Phys. 12, 166-237.

[14] Svergun, D.I. (1992) Determination of the regularisation parameter in indirecttransform methods using perceptual criteria. J. Appl. Crystallogr. 25, 495-503.

[15] Svergun, D.I., Barberato, C. and Koch, M.H.J. (1995) CRYSOL - a program to evaluate X-ray solution scattering of biological macromolecules from atomic coordinates. J. Appl. Crystallogr. 28, 768-773.

[16] Steinberg, I.Z. (1971) Long-range nonradiative transfer of electronic excitation energy in proteins and polypeptides. Annu Rev Biochem 40, 83-114.

[17] Wallon, G., Kryger, G., Lovett, S.T., Oshima, T., Ringe, D. and Petsko, G.A. (1997) Crystal structures of Escherichia coli and Salmonella typhimurium 3-isopropylmalate 
dehydrogenase and comparison with their thermophilic counterpart from Thermus thermophilus. J Mol Biol 266, 1016-31.

[18] Imada, K., Inagaki, K., Matsunami, H., Kawaguchi, H., Tanaka, H., Tanaka, N. and Namba, K. (1998) Structure of 3-isopropylmalate dehydrogenase in complex with 3isopropylmalate at 2.0 A resolution: the role of Glu88 in the unique substraterecognition mechanism. Structure 6, 971-82.

[19] Hurley, J.H. and Dean, A.M. (1994) Structure of 3-isopropylmalate dehydrogenase in complex with NAD+: ligand-induced loop closing and mechanism for cofactor specificity. Structure 2, 1007-16.

[20] Kadono, S., Sakurai, M., Moriyama, H., Sato, M., Hayashi, Y., Oshima, T. and Tanaka, N. (1995) Ligand-induced changes in the conformation of 3-isopropylmalate dehydrogenase from Thermus thermophilus. J Biochem (Tokyo) 118, 745-52. 


\section{LEGENDS to the FIGURES}

Fig. 1 FRET spectra characteristic of $T t(A)$ and $E c$ (B) IPMDHs. Fluorescence emission spectra were recorded with both $T t$ (A) and $E c$ (B) IPMDHs (0.32 $\mu \mathrm{M}$ monomers) upon excitation at $295 \mathrm{~nm}$. In both cases the dotted lines represent spectra of complexes of IPMDH with NADH, which are the sum of the separate spectra of the protein (dashed lines) and of NADH (dash-dot-dash). Addition of $0.5 \mathrm{mM}$ IPM (in the presence of $1 \mathrm{mM}$ EDTA) does not cause any appreciable changes in this spectrum (dotted lines). However, addition of MgIPM at saturation causes appearance of the characteristic FRET spectrum (continuous lines).

Fig. 2 Time courses of denaturation of $E c$ IPMDH as followed by measuring changes of protein fluorescence (A) and thiol-reactivity (B). Experimental details of denaturation of $E c$ IPMDH is described in Methods. In case of fluorimetric detection (A) $0.32 \mu \mathrm{M}$ monomers were present in the cuvette in the absence of any additives (dashed line) and in the presence of $1 \mathrm{mM}$ IPM plus $1 \mathrm{mM} \mathrm{MnCl}_{2}$ (solid line), $10 \mathrm{mM}$ IPM plus $10 \mathrm{mM}$ EDTA (dotted line) or 1 $\mathrm{mM} \mathrm{MnCl}_{2}$ (dash-dot-dash). By detecting liberation of the thiol-groups (B) $11.2 \mu \mathrm{M}$ monomers were present in the absence (dashed line) and in the presence of $3 \mathrm{mM}$ NAD or NADH (dash-dot-dash), $10 \mathrm{mM}$ IPM plus $10 \mathrm{mM}$ EDTA (dotted line) as well as in the presence of $1 \mathrm{mM}$ IPM and $1 \mathrm{mM} \mathrm{MnCl}$ (solid line). The complexes with MnIPM plus either 3 mM NAD (functioning complex) or NADH (non-functioning complex) provided time courses identical with the solid line. Under native conditions, the modification curve of the fast-reacting thiols ( 1 mole/mole subunit) is also plotted (dash-dot-dash).

Fig. 3 Comparison of the experimental the calculated SAXS scattering curves for $T t$ IPMDH The experiments were carried out both in the presence of either $\mathrm{MnCl}_{2}$ or $\mathrm{MgCl}_{2}(2 \mathrm{~b}$ 
only) (A) and in the absence of the metal-ion, i.e. in the presence of EDTA (B). In the case (A) the apoenzyme (1), the complexes with IPM (2a for $\mathrm{MnCl}_{2}$ and $2 \mathrm{~b}$ for $\mathrm{MgCl}_{2}$ ), with NAD (3), with NADH (4), as well with IPM plus NAD (5) and with IPM plus NADH (6) have been investigated. In the case (B) the apoenzyme (1), the complexes with IPM (2), with IPM plus NAD (3) and with IPM plus NADH (4) have been investigated. The protein and ligand concentrations of the samples are given in the Methods. The logarithm of the scattering intensity $(I)$ is plotted as a function of the momentum transfer $s$. Dots (with error bars): experimental data, solid lines: best fits from the models generated from the crystallographic data (cf. Methods and Table 2). The successive curves are displaced down by one logarithmic unit for better visualization.

Fig. 4 A possible role of the metal-ion in the stereospecific substrate-like binding of IPM. The ribbon diagrams of apo $T t$ IPMDH (gray, PDB code 2Y3Z) and its complex with MnIPM (black, PDB code 2Y41) are shown after superimposition of the $\beta$-sheets F. The bound MnIPM molecule is represented by ball-and-stick model (coloured by atoms) and the side chains are illustrated by stick models. Dashed lines represent atomic interactions. The sixth coordinating ligand of $\mathrm{Mn}^{2+}$ is the side-chain of D217 from the other subunit that is not illustrated here. 
Table 1. Effect of substrates on the denaturation rate of $E c$ IPMDHs. Denaturation of $E c$ IPMDH was followed by two different methods: fluorimetry and thiol reactivity as described in the Methods.

\begin{tabular}{ccc}
\hline \multirow{2}{*}{ Additives } & \multicolumn{2}{c}{ Rate constants of Denaturation $\left(\mathrm{min}^{-1}\right)$} \\
\cline { 2 - 3 } No & Fluorimetric detection & Detection by thiol-reactivity \\
Mn $^{2+}$ & $0.201 \pm 0.07$ & $0.195 \pm 0.03$ \\
MnIPM & $0.110 \pm 0.04$ & $0.176 \pm 0.03$ \\
Mn $^{2+}$ and NAD & $0.017 \pm 0.005$ & $0.042 \pm 0.004$ \\
Mn $^{2+}$ and NADH & n.d. & $0.154 \pm 0.04$ \\
MnIPM and NAD & n.d. & $0.178 \pm 0.04$ \\
MnIPM and NADH & n.d. & $0.044 \pm 0.005$ \\
EDTA & n.d. & $0.043 \pm 0.004$ \\
EDTA and IPM & $0.201 \pm 0.07$ & $0.203 \pm 0.04$ \\
EDTA, IPM and NAD & $0.184 \pm 0.06$ & $0.204 \pm 0.05$ \\
EDTA, IPM and NADH & n.d. & $0.177 \pm 0.03$ \\
\hline
\end{tabular}


Table 2. Comparison of SAXS experimental data with those derived from the crystallographic models.

\begin{tabular}{|c|c|c|c|c|c|c|c|}
\hline \multicolumn{3}{|c|}{ SAXS experiments } & \multicolumn{3}{|c|}{$\begin{array}{c}\text { Discrepancy values } \chi \text { (calculated from Eq.(1)) } \\
\text { between the scattering from crystallographic } \\
\text { models and experimental data }\end{array}$} & \multicolumn{2}{|c|}{$\begin{array}{l}\text { Volume fractions of } \\
\text { open/closed structures }\end{array}$} \\
\hline \multirow{2}{*}{ Additives } & \multicolumn{2}{|c|}{$\mathrm{Rg}, \AA^{\mathrm{a}}$} & \multirow{2}{*}{$\begin{array}{c}\text { Closed } \\
\text { crystal } \\
\text { structure } \\
\end{array}$} & \multirow{2}{*}{$\begin{array}{c}\text { Open } \\
\text { crystal } \\
\text { structure }\end{array}$} & \multirow[t]{2}{*}{$\begin{array}{l}\text { Open/closed } \\
\text { mixture }\end{array}$} & \multirow[t]{2}{*}{$\mathrm{V}_{\text {closed, }} \%$} & \multirow[t]{2}{*}{$\mathrm{V}_{\text {open, }} \%$} \\
\hline & $\begin{array}{l}\text { GNOM } \\
\text { method }\end{array}$ & $\begin{array}{l}\text { Guinier } \\
\text { method }\end{array}$ & & & & & \\
\hline No substrate & $28.6 \pm 0.2$ & $28.7 \pm 0.3$ & 2.21 & 1.08 & 1.08 & 0 & 100 \\
\hline $\mathrm{Mn}^{2+}$ & $28.5 \pm 0.2$ & $28.6 \pm 0.3$ & 2.29 & 1.15 & 1.15 & 0 & 100 \\
\hline MnIPM & $27.5 \pm 0.2$ & $27.6 \pm 0.3$ & 1.28 & 1.36 & 1.09 & $55 \pm 5$ & $45 \pm 5$ \\
\hline MgIPM & $27.6 \pm 0.2$ & $27.7 \pm 0.3$ & 1.41 & 1.45 & 1.07 & $50 \pm 5$ & $50 \pm 5$ \\
\hline $\mathrm{Mn}^{2+}$ and NAD & $28.2 \pm 0.2$ & $28.3 \pm 0.3$ & 1.80 & 1.09 & 1.04 & $10 \pm 5$ & $90 \pm 5$ \\
\hline $\mathrm{Mn}^{2+}$ and NADH & $28.1 \pm 0.2$ & $28.2 \pm 0.3$ & 1.74 & 1.06 & 1.01 & $12 \pm 5$ & $88 \pm 5$ \\
\hline MnIPM and NAD & $28.1 \pm 0.2$ & $28.2 \pm 0.3$ & 1.65 & 1.06 & 1.03 & $10 \pm 5$ & $90 \pm 5$ \\
\hline MnIPM and NADH & $27.5 \pm 0.2$ & $27.6 \pm 0.3$ & 1.47 & 1.78 & 1.08 & $55 \pm 5$ & $45 \pm 5$ \\
\hline EDTA & $28.4 \pm 0.2$ & $28.4 \pm 0.3$ & 2.10 & 1.07 & 1.07 & 0 & 100 \\
\hline EDTA and IPM & $28.4 \pm 0.2$ & $28.4 \pm 0.3$ & 2.16 & $\mathbf{1 . 0 3}$ & 1.03 & 0 & 100 \\
\hline EDTA, IPM and NAD & $28.3 \pm 0.2$ & $28.4 \pm 0.3$ & 2.18 & 1.05 & 1.05 & 0 & 100 \\
\hline EDTA, IPM and NADH & $28.3 \pm 0.2$ & $28.4 \pm 0.3$ & 2.27 & 1.04 & 1.04 & 0 & 100 \\
\hline \multicolumn{3}{|c|}{$\operatorname{Rg}$ (theoretical), angstroms ${ }^{b}$} & 26.67 & 28.45 & & & \\
\hline \multicolumn{3}{|c|}{ Molecular mass, $\mathrm{kDa}^{\mathrm{b}}$} & 74.3 & 73.8 & & & \\
\hline
\end{tabular}

${ }^{\mathrm{a}}$ The $\mathrm{R}_{\mathrm{g}}$ values were computed by two alternative methods, using the program GNOM and Guinier approximation, respectively.

${ }^{\mathrm{b}}$ Radius of gyration and molecular mass of the high resolution models as retrieved from the PDB.

The PDB codes: 2Y41 for the closed crystal structure of the complex of $T t$ IPMDH with MnIPM, 1XAA for the open crystal structure of apo $T t$ IPMDH.

${ }^{\mathrm{c}}$ The fits for open/closed mixture were obtained by OLIGOMER, $\mathrm{V}_{\text {open }}$ and $\mathrm{V}_{\text {closed }}$ correspond to the volume fractions of each state found by OLIGOMER.

The minimum values of discrepancy (in bold) indicate the best correlation between SAXS data and crystallographic model.

The small variations in the calculated molecular mass are due to different numbers of residues resolved in different crystal structures. 

A

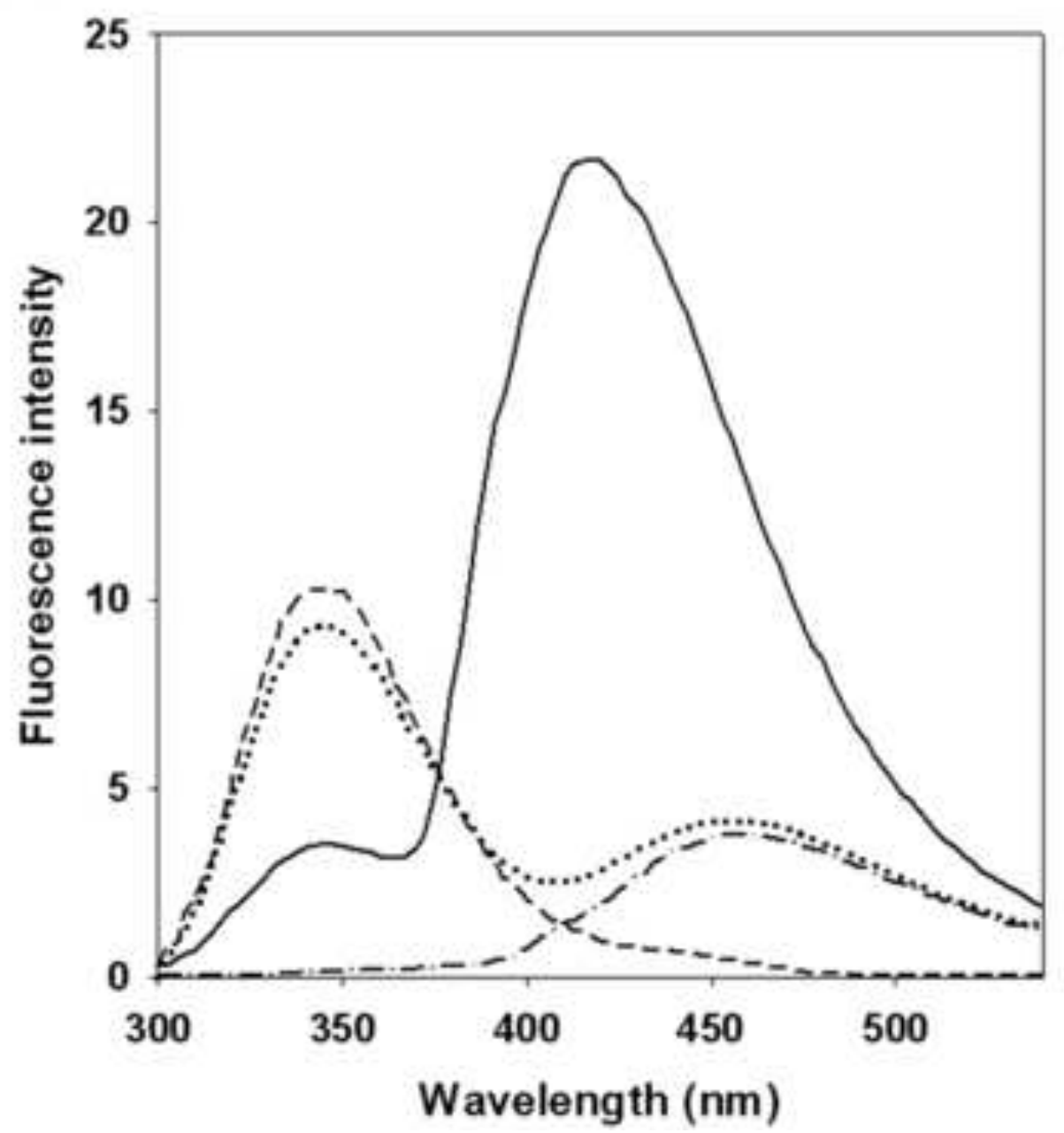

B

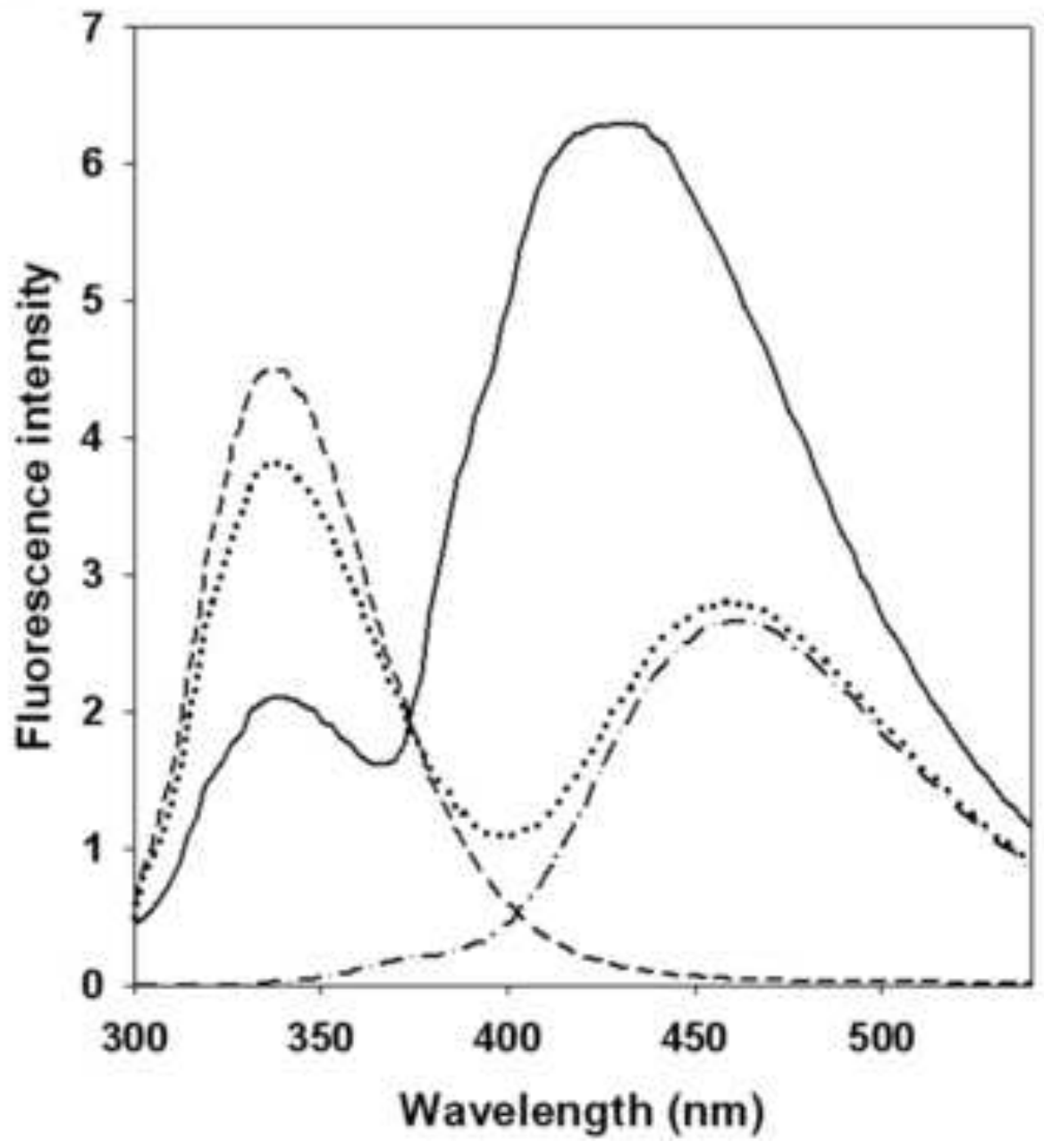



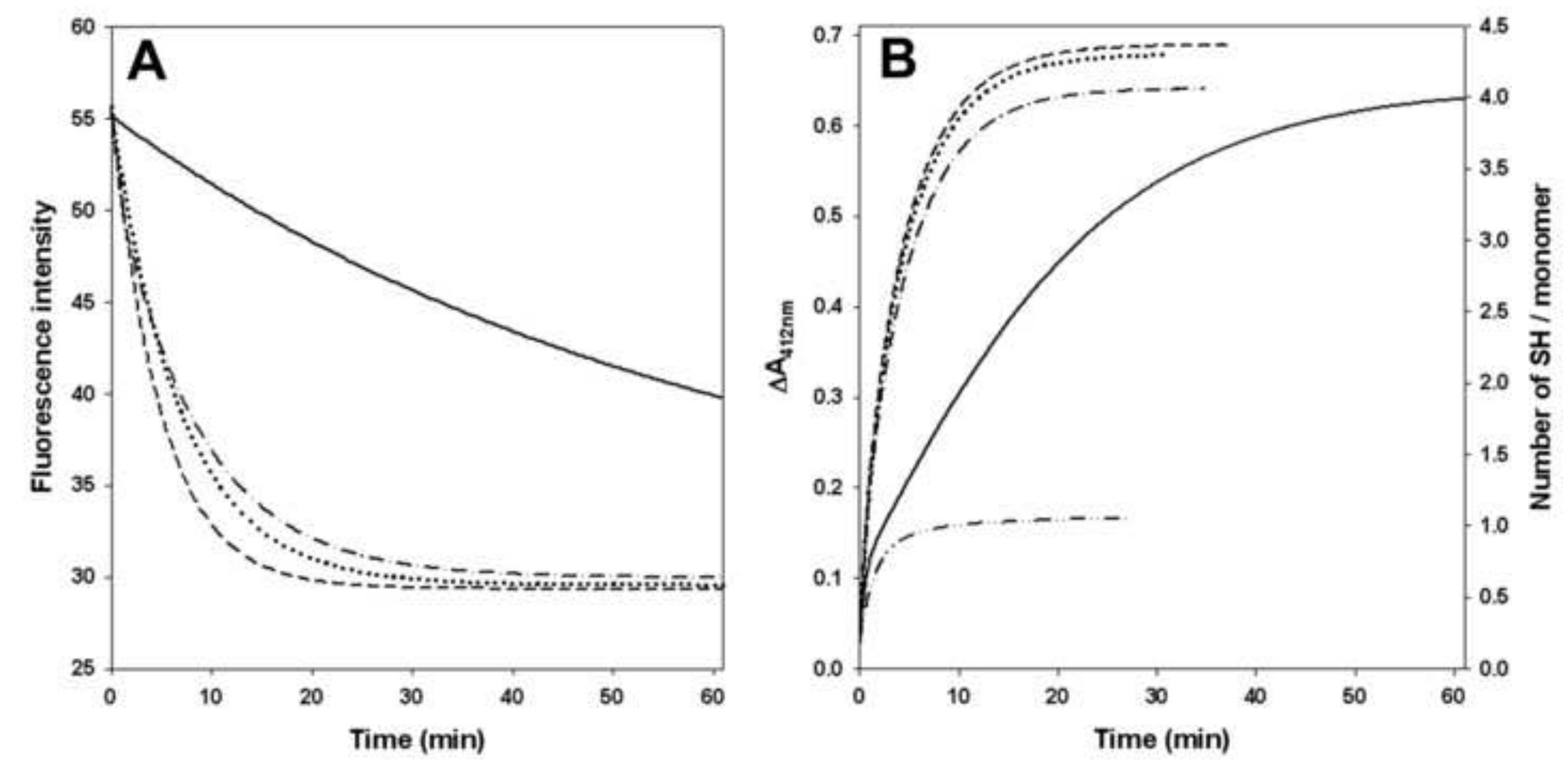

है


A Igl, relative

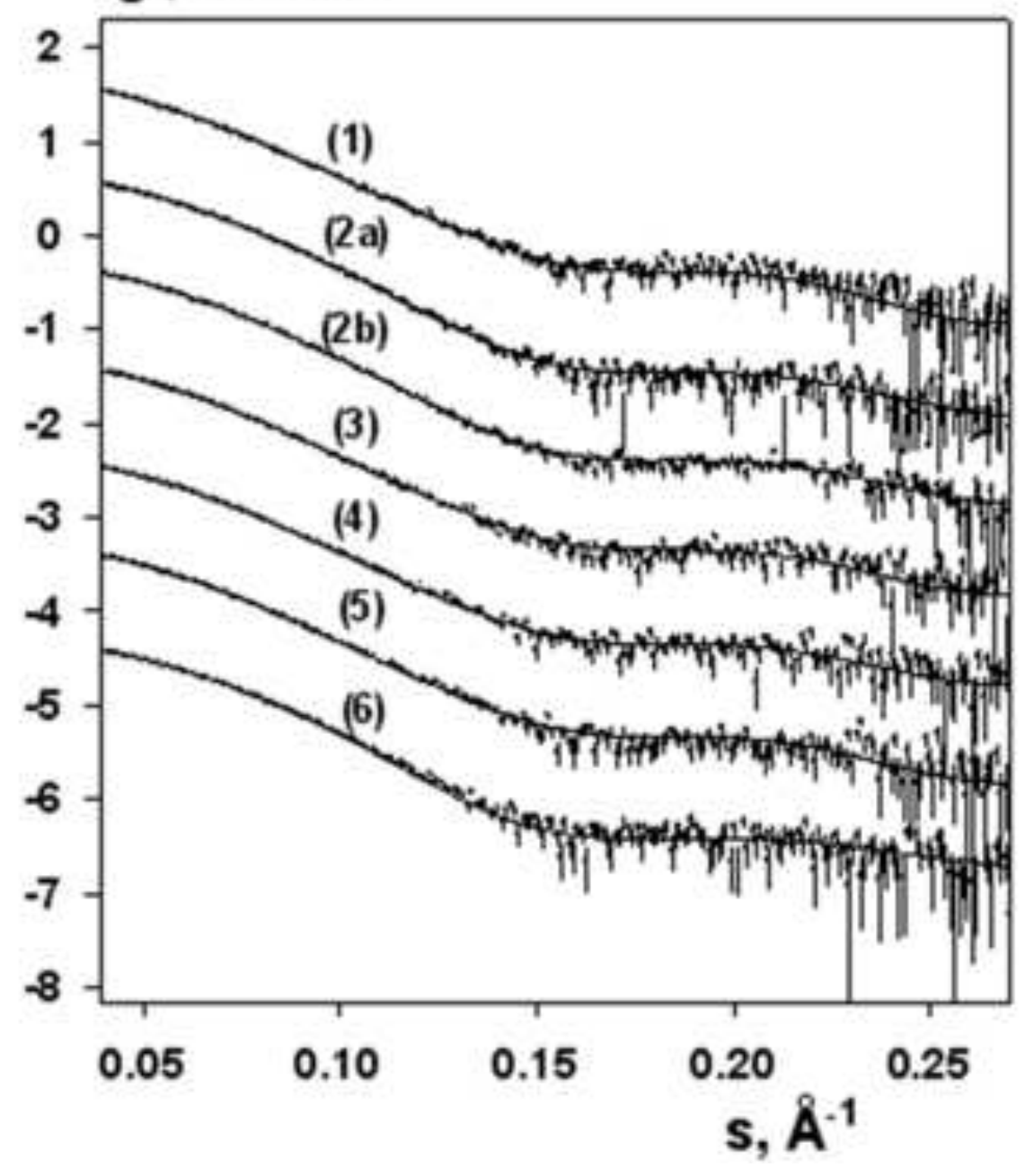

\section{B Igl, relative}

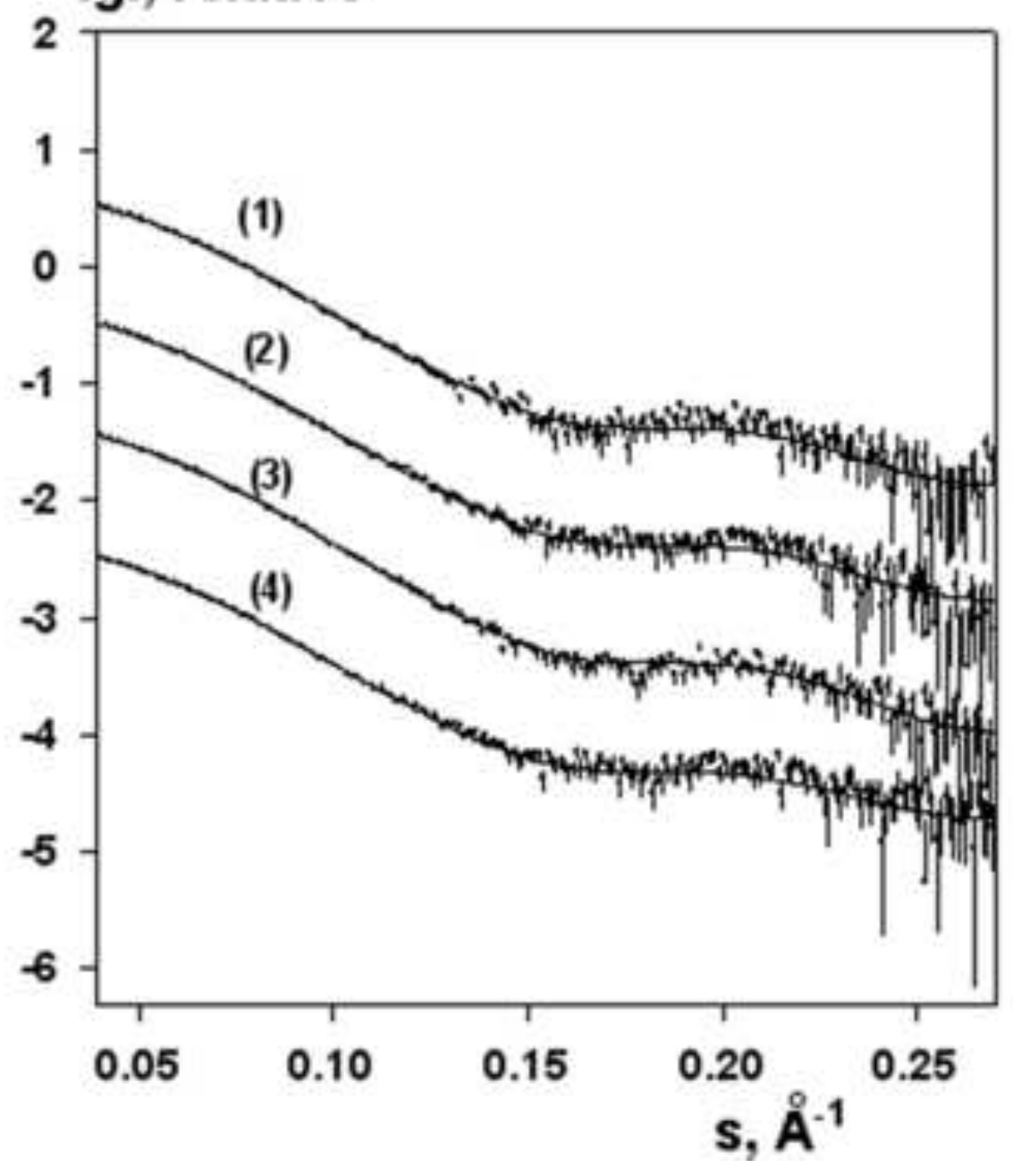




\section{$\beta$-strand G}

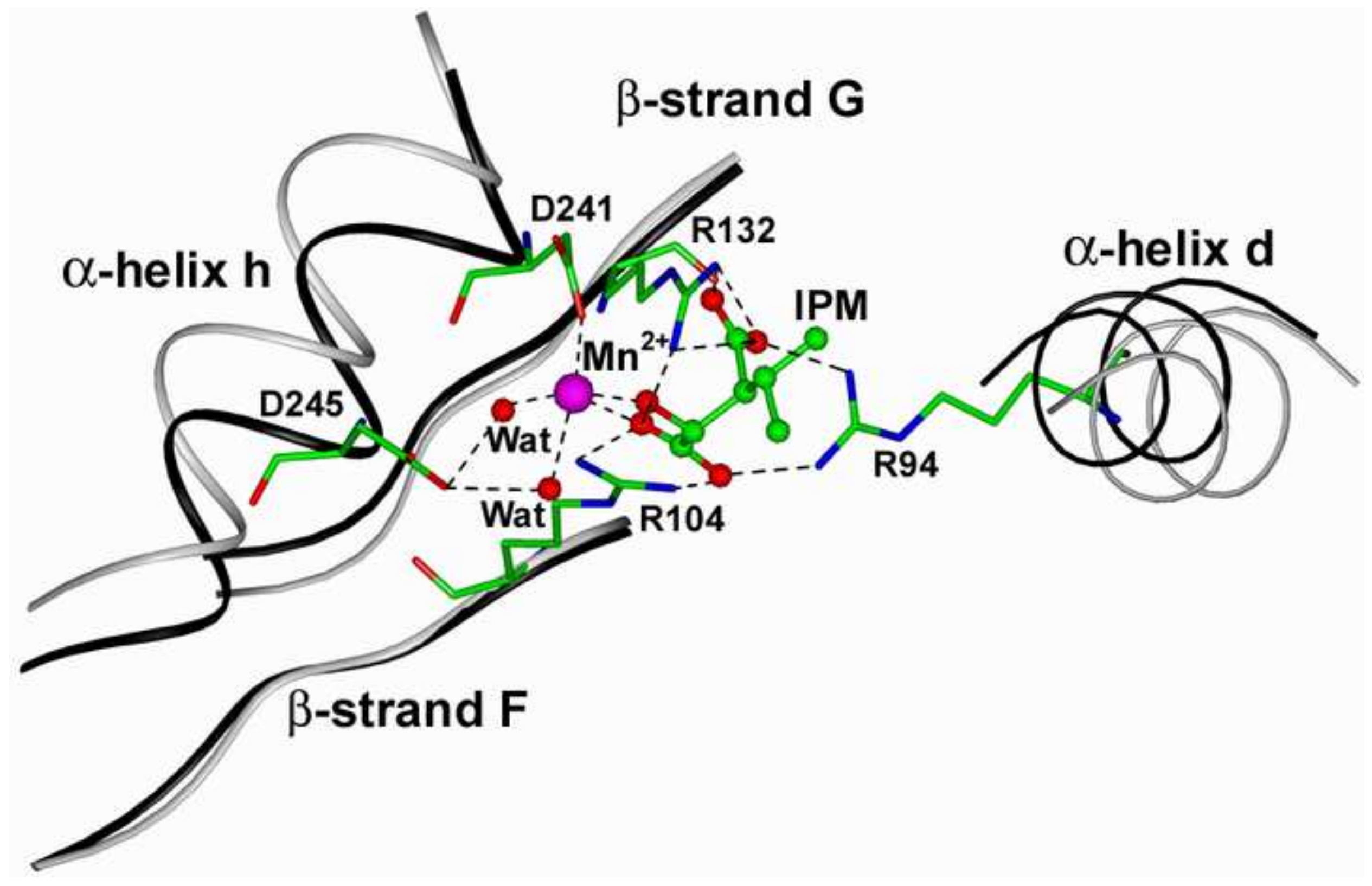

